\title{
Association of Serum Nonesterified Fatty Acids with Cardiovascular Event in Patients with Chronic Kidney Disease
}

\author{
Chentang $\mathrm{Wu} \mathbb{1 D}^{\prime}$ \\ Xueyun Chen ${ }^{2}$ \\ 'Department of Cardiovascular Medicine, \\ Mindong Hospital of Fujian Medical \\ University, Fuan, Fujian, 355000, People's \\ Republic of China; ${ }^{2}$ Department of \\ Endocrinology, Mindong Hospital of \\ Fujian Medical University, Fuan, Fujian, \\ 355000, People's Republic of China
}

Background: Chronic kidney disease (CKD) has been suggested to be associated with a high risk of cardiovascular diseases (CVD). The study aimed to evaluate the prognostic significance of nonesterified fatty acid (NEFA), also well known as free fatty acid, on predicting cardiovascular events in patients with CKD.

Methods: A total of 957 hospitalized patients with CKD in a stable clinical condition were enrolled at baseline. Then, the serum NEFA levels were measured. These included patients were prospectively followed up for a median of 10.2 years (range $=0.4-11.5$ years). We assessed whether serum NEFA levels at baseline can predict cardiovascular event during the follow-up.

Results: A total of 278 (29.1\%) patients experienced cardiovascular events during followup. The Kaplan-Meier curve demonstrated that patients with higher serum NEFA levels $(\geq 19.8 \mathrm{mg} / \mathrm{dl})$ had a higher rate of cardiovascular events than patients with lower NEFA levels $(<19.8 \mathrm{mg} / \mathrm{dl})$. Multivariate Cox regression analysis suggested that elevated serum NEFA levels $(\mathrm{HR}=1.62 ; 95 \% \mathrm{CI} 1.40-2.16, \mathrm{P}<0.001)$ were independently associated with increased risk of cardiovascular events after correction for clinical confounding factors.

Conclusion: Elevated serum NEFA levels were associated with higher risk of cardiovascular events and may be a new parameter predicting cardiovascular events in patients with $\mathrm{CKD}$, which may strengthen its potential effect in clinical practice.

Keywords: nonesterified fatty acid, cardiovascular event, chronic kidney disease, KaplanMeier, Cox regression

\section{Introduction}

The incidence rate and mortality of chronic kidney diseases (CKD) are increasing year by year, causing serious financial burden and threatening human life and health. ${ }^{1-3}$ CKD patients are at high risk of cardiovascular disease (CVD) and the CVD is a major cause of death in CKD patients, accounting for about $50 \%$ of total mortality in CKD patients. ${ }^{4-6}$ Oxidative stress and inflammation play an important role in this pathological process. ${ }^{7-9}$ Effective prediction and/or prevention of cardiovascular events is of great significance for CKD patients.

It is well known that nonesterified fatty acid (NEFA) is free fatty acid that originates from hydrolysis of triglycerides in adipose tissue. ${ }^{10}$ NEFA is an important energy substance in many tissues and excessive accumulation and metabolites of NEFA can be harmful, especially in myocardial tissue. ${ }^{11,12}$ Many mechanistic evidence has been used to report that NEFA is associated with CVD risk, obesity and type 2 diabetes mellitus
Department of Endocrinology, Mindong Hospital of Fujian Medical University, No. 89 Heshan Road, Fuan City, 355000,

Fujian Province, People's Republic of China

Email chenxueyun8888@I26.com 
and provides a across association among these diseases. ${ }^{13}$ Interestingly, existing epidemiologic studies suggested that NEFA contributed to increased risk of cardiovascular events such as myocardial infarction and CVD death in the general population. ${ }^{14-17}$ However, some other studies did not find any significant association. ${ }^{18-20}$ The difference in results may be explained by different study designs used, different populations selected, different hypotheses investigated and different confounding factors adjusted.

Until now, few studies evaluated the association in a CKD population. In the present study, we included a large number of samples in a population with CKD and investigated whether serum NEFA levels were related to increased risk of composite endpoint (cardiovascular events), including myocardial infarction, ischemic and/or hemorrhagic stroke, worsening heart failure, peripheral vascular diseases and sudden cardiac death during the follow-up of 10 years. We hope to provide a strong evidence to resolve previous controversies.

\section{Materials and Methods}

\section{Study Population}

A total of 957 individuals were identified as having CKD from Mindong Hospital of Fujian Medical University due to worsening CKD between April 2008 and July 2010. Before admission, these CKD patients have been in a stable clinical condition for more than 6 months. CKD is defined as chronic renal structure and dysfunction caused by various reasons including abnormal eGFR (< $60 \mathrm{~mL} / \mathrm{min} \cdot 1.73 \mathrm{~m}^{2}$ ), pathological damage, abnormal blood or urine composition or abnormal imaging examination for more than 3 months. ${ }^{21}$ For research purposes of the study, patients with eGFR $<60\left[\mathrm{~mL} / \mathrm{min}\right.$ per $\left.1.73 \mathrm{~m}^{2}\right]$ and/or having $\mathrm{CKD}$ history were included $(\mathrm{N}=957)$. The measurement of serum NEFA was performed in all included patients with CKD for analysis. Some CKD patients with serious liver diseases, cancers or other serious diseases were excluded ( $\mathrm{N}=56$ ). All included patients with $\mathrm{CKD}$ were treated with regular therapy during hospitalization and were followed up after discharge. Patients with a CVD history $(\mathrm{N}=56)$ included diabetes mellitus, heart failure, stroke, myocardial infarction and others. Additionally, CKD is mainly classified from stage I to stage $V$ according to eGFR: ${ }^{21}$ stage $\mathrm{I}, \geq 90 \mathrm{~mL} / \mathrm{min} \cdot 1.73 \mathrm{~m}$ 2; stage II, $60-90 \mathrm{~mL} / \mathrm{min} \cdot 1.73 \mathrm{~m}^{2}$; stage III, $30-59 \mathrm{~mL} /$ $\min \cdot 1.73 \mathrm{~m}^{2}$; stage IV, $15-29 \mathrm{~mL} / \mathrm{min} \cdot 1.73 \mathrm{~m}^{2}$; stage IV, $<15 \mathrm{~mL} / \mathrm{min} \cdot 1.73 \mathrm{~m}^{2}$.
According to Declaration of Helsinki guidelines, the Ethics Committee of Mindong Hospital of Fujian Medical University approved this study and all patients in our study gave written informed consent.

\section{Follow-Up}

CKD patients were followed up by telephone and/or reviewing the medical records 3 times every year until the occurrence of endpoint events. The endpoints of the study were defined as cardiovascular events. Adequate collection of endpoint events was ensured by our continuous and reliable surveillance system. If current status of CKD patients was unknown, referring cardiologists and/or general practitioners were contacted and invited to identify the patient's condition. For the purpose of this study, the cardiovascular events were defined as myocardial infarction $(\mathrm{N}=92)$, ischemic and/or hemorrhagic stroke $(\mathrm{N}=88)$, worsening heart failure $(\mathrm{N}=73)$, peripheral vascular diseases $(\mathrm{N}=13)$ and sudden cardiac death $(\mathrm{N}=12)$. Thirty-two patients with CKD were lost during the follow-up period.

\section{Covariants}

This study was carried out under standardized conditions. Body mass index (BMI) is weight (kg) divided by height square $\left(\mathrm{cm}^{2}\right)$. Smoking status was defined as "current smoker" or "not current smoker". Drinking status was defined as "current drinker" or "not current drinker". Regular physical activity was defined as "frequency of regular physical activity $\geq 3$ weekly or not". The measure of blood pressure (BP) was performed in the supine position at rest for a mean value of two times that of the right arm. "Fasting blood glucose (FBG) $\geq 126 \mathrm{mg} / \mathrm{dl}$ " or "taking diabetes medications" was defined as diabetes mellitus. "Medications" was defined as "yes" or "no".

\section{Laboratory Measurements}

Venous blood samples were collected in the first morning admission and stored at $-80^{\circ} \mathrm{C}$ until required for analyses. Serum NEFA levels were measured by a NEFA kit (Wako Chemicals, Neuss, Germany). The coefficient of variation in this measurement is about $5 \% .{ }^{19} \mathrm{C}$-reactive protein (CRP) was tested by using a Behring BN ProSpec analyzer (Dade Behring). The CKD Epidemiology Collaboration (CKDEPI) creatinine was used to calculate eGFR. ${ }^{22}$ Measuring FBG was performed by the enzymatic-immunologic assay (Gluc-DH; Merck, Darmstadt, Germany). The total cholesterol, low-density lipoprotein cholesterol (LDL), highdensity lipoprotein cholesterol (HDL) and triglycerides 
with the use of the Siemens ADVIA 2400 automatic biochemistry analyzer (Siemens AG). The N-terminal prohormone of B-type natriuretic peptide (NT-proBNP), albumin (ALB) and hemoglobin ( $\mathrm{Hb}$ ) was measured by using an automated blood counter (Sysmex XE5000; Emilio de Azevedo Campos, Porto, Portugal).

\section{Statistical Analyses}

All the data were analyzed by using SPSS 26.0. $P \leq 0.05$ was considered to be statistically significant. KolmogorovSmirnov test was used to analyze the normality of the data. Data that were not normally distributed were analyzed by the Mann-Whitney $U$-test and then were expressed as the median (interquartile range [IQR]). Data were analyzed by independent $t$-test and presented as the mean \pm SD for normally distributed data. The chi-square test was used to analyze categorical variables and was expressed as n (\%). Baseline characteristics upon entry into the cohort were compared between tertiles of serum NEFA levels. In multivariate analysis, adjustment of confounding factors including age and gender was made in Model 1. Adjustment of confounding factors including age, gender, BMI, current smoker, current drinker, systolic and diastolic BP and regular physical activity was made in Model 2. In Model 3, adjustments of age, gender, BMI, current smoker, current drinker, systolic and diastolic BP, regular physical activity, stage of CKD, laboratory indexes and medications were made.

Serum levels of serum NEFA levels at baseline were categorized by quartiles (quartile $1: \leq 25$ th percentile; quartile 2: 25 th to 50 th percentile; quartile 3 : 50th to 75 th percentile; quartile $4: \geq 75$ th percentile). Cox regression analysis was performed to identify the independent predictive value of serum NEFA levels for cardiovascular events in CKD patients. To further evaluate the independent association, we further excluded the effect of "dialysis treatment" by sensitivity analysis. Finally, we also analyzed the association between serum NEFA levels at baseline and cardiovascular events during the follow-up by stratified analysis. Cardiovascular event-free curve was constructed by the Kaplan-Meier method and Log rank test was performed.

\section{Results}

\section{Clinical Characteristics of COPD Patients}

\section{at Baseline ( $N=957)$}

The clinical features of CKD patients are described in Table 1. All included patients with CKD were divided into two groups according to the median value of serum NEFA levels $(19.8 \mathrm{mg} / \mathrm{dl})$. CKD patients with higher levels of serum IL-10 ( $\geq 19.8 \mathrm{mg} / \mathrm{dl})$ had higher rates of cardiovascular events than those who had lower levels of serum IL-10 $(<19.8 \mathrm{mg} / \mathrm{dl})$, which initially suggested that elevated NEFA levels were potentially associated with increased risk of cardiovascular events [201 (42.0\%) VS 77 (16.1\%)]. Furthermore, CKD patients with high serum NEFA levels had higher BMI, systolic and diastolic BP and higher rates of CVD history and dialysis treatment, and tended to be current smoker, current drinker and less physical activity. As for laboratory indexes and medications, the information was also described in detail (Table 1).

\section{Elevated Serum Levels of NEFA Were Independently Associated with Increased Risk of Cardiovascular Events in CKD Patients ( $\mathrm{N}=957)$}

All included CKD patients were prospectively followed up for a median of 10.2 (range $=0.4-11.5$ ) years. Cardiovascular events occurred in 278 of CKD patients included myocardial infarction ( $\mathrm{N}=92)$, ischemic and/or hemorrhagic stroke $(\mathrm{N}=88)$, worsening heart failure $(\mathrm{N}=73)$, peripheral vascular diseases $(\mathrm{N}=13)$ and sudden cardiac death $(\mathrm{N}=12)$ during the follow-up period. Kaplan-Meier analysis suggested that CKD patients with serum NEFA levels above median $(19.8 \mathrm{mg} / \mathrm{dl})$ had a higher rate of cardiovascular events than those with serum NEFA levels below the median value (Log rank test, $P<0.001$, Figure 1). To furthermore evaluate the association of serum NEFA levels with cardiovascular events in CKD patients, a multivariate Cox proportional hazard regression model was performed. Cox analysis revealed that elevated serum NEFA levels were independent and significant with increased risk of cardiovascular events (HR=1.62, 95\% CI 1.40-2.16, P-trend $<0.001$, Table 2) after adjustments of age, gender, BMI, current smoker, current drinker, systolic and diastolic BP, regular physical activity, stage of CKD, laboratory indexes and medications were made in Model 3.

We further clarify the association between serum NEFA levels and cardiovascular events in patients with CKD. An additional sensitivity analysis was performed by adding "dialysis treatment" as a covariate. Our results still showed that elevated serum NEFA levels were significantly associated with a higher risk of cardiovascular death $(\mathrm{HR}=1.46$, 
Table I Clinical Characteristics of CKD Patients at Baseline (N=957)

\begin{tabular}{|c|c|c|c|}
\hline Characteristics & NEFA $<19.8 \mathrm{mg} / \mathrm{dl}(\mathrm{N}=478)$ & NEFA $\geq 19.8 \mathrm{mg} / \mathrm{dl}(\mathrm{N}=479)$ & P value \\
\hline Age $(Y)$ & $75.3 \pm 11.6$ & $76.9 \pm 12.4$ & 0.156 \\
\hline Gneder (male), n (\%) & $302(63.2)$ & $288(60.1 .4)$ & 0.174 \\
\hline BMI $\left(\mathrm{kg} / \mathrm{m}^{2}\right)$ & $30.3 \pm 5.7$ & $32.8 \pm 6.5$ & $<0.001$ \\
\hline Smoking status (current smoker), n (\%) & $127(26.6)$ & $172(35.9)$ & 0.010 \\
\hline Drinking status (current drinker), $\mathrm{n}$ (\%) & $101(2 I . I)$ & $146(30.5)$ & 0.012 \\
\hline Systolic BP (mmHg) & $153.7 \pm 15.2$ & $161.4 \pm 18.9$ & $<0.001$ \\
\hline Diastolic BP (mmHg) & $85.5 \pm 8.9$ & $94.6 \pm 9.5$ & $<0.001$ \\
\hline Regular physical activity ( $\geq 3$ weekly), n (\%) & $192(40.1))$ & $149(31.2)$ & 0.005 \\
\hline Cardiovascular events during follow-up, n (\%) & $77(16.1)$ & $201(42.0)$ & $<0.001$ \\
\hline Dialysis treatment, $\mathrm{n}(\%)$ & $74(15.5)$ & $159(33.2)$ & $<0.001$ \\
\hline \multicolumn{4}{|l|}{ CVD history } \\
\hline Myocardial infarction, n (\%) & $2(0.4)$ & $12(2.5)$ & 0.003 \\
\hline Ischemic and/or hemorrhagic stroke, $n$ (\%) & $7(1.5)$ & $14(2.9)$ & 0.002 \\
\hline Heart failure, n (\%) & II (2.3) & $20(4.2)$ & $<0.001$ \\
\hline Diabetes mellitus, n (\%) & $34(7.1)$ & $52(10.9)$ & $<0.001$ \\
\hline Others, n (\%) & $2(0.4)$ & $5(1.0)$ & 0.011 \\
\hline \multicolumn{3}{|l|}{ Stage of CKD } & 0.006 \\
\hline CKD I, n (\%) & $77(16.1)$ & $0(0)$ & \\
\hline CKD II, n (\%) & $178(37.2)$ & $45(9.4)$ & \\
\hline CKD III, n (\%) & $165(34.5)$ & $201(42.0)$ & \\
\hline CKD IV, n (\%) & $38(7.9)$ & $39(8.1)$ & \\
\hline CKD V, n (\%) & $20(4.2)$ & $194(40.5)$ & \\
\hline \multicolumn{4}{|l|}{ Laboratory indexes } \\
\hline C-reactive protein (mg/L) & $7.8(3.9-15.3)$ & I5.3 (7.6-24.) & $<0.001$ \\
\hline NT-proBNP (pg/mL) & $335.2(102.4-457.4)$ & $456.3(145.8-684.8)$ & $<0.001$ \\
\hline Albumin $(g / d l)$ & $4.8 \pm 0.9$ & $3.5 \pm 0.6$ & $<0.001$ \\
\hline eGFR (mL/min per $1.73 \mathrm{~m} 2)$ & $54.8 \pm 9.3$ & $43.5 \pm 8.5$ & $<0.001$ \\
\hline FBG (mg/dl) & $85.3 \pm 12.4$ & $92.7 \pm 14.5$ & $<0.001$ \\
\hline $\mathrm{Hb}(\mathrm{mg} / \mathrm{L})$ & $122 \pm 7.9$ & $100 \pm 5.7$ & $<0.001$ \\
\hline Total cholesterol (mg/dl) & $227.5 \pm 36.4$ & $249.6 \pm 38.4$ & $<0.001$ \\
\hline Triglycerides (mg/dl) & $136.9 \pm 12.3$ & $152.6 \pm 14.4$ & $<0.001$ \\
\hline $\mathrm{HDL}(\mathrm{mmol} / \mathrm{L})$ & $46.4 \pm 15.7$ & $40.3 \pm 13.2$ & $<0.001$ \\
\hline LDL (mmol/L) & $135.3 \pm 24.5$ & $149.4 \pm 25.6$ & $<0.001$ \\
\hline \multicolumn{4}{|l|}{ Medications } \\
\hline ACE inhibitor or ARB (\%) & $79(16.5)$ & $125(26.1)$ & 0.019 \\
\hline Calcineurin inhibitor (\%) & $54(11.3)$ & $75(15.7)$ & 0.034 \\
\hline Diuretic, n (\%) & $102(21.3)$ & I $12(23.4)$ & 0.689 \\
\hline
\end{tabular}

Note: Data are presented as mean \pm SD for normally distributed data, as median (interquartile range) for nonnormally distributed data, and as $\mathrm{n}$ (\%) for categoric variables. Abbreviations: CKD, chronic kidney disease; BMI, body mass index; BP, blood pressure; CVD, cardiovascular disease; NT-proBNP, N-terminal prohormone of B-type natriuretic peptide; eGFR, estimated glomerular filtration rate; FBG, fasting blood glucose; Hb, hemoglobin; HDL, high density lipoprotein; LDL, low density lipoprotein. 


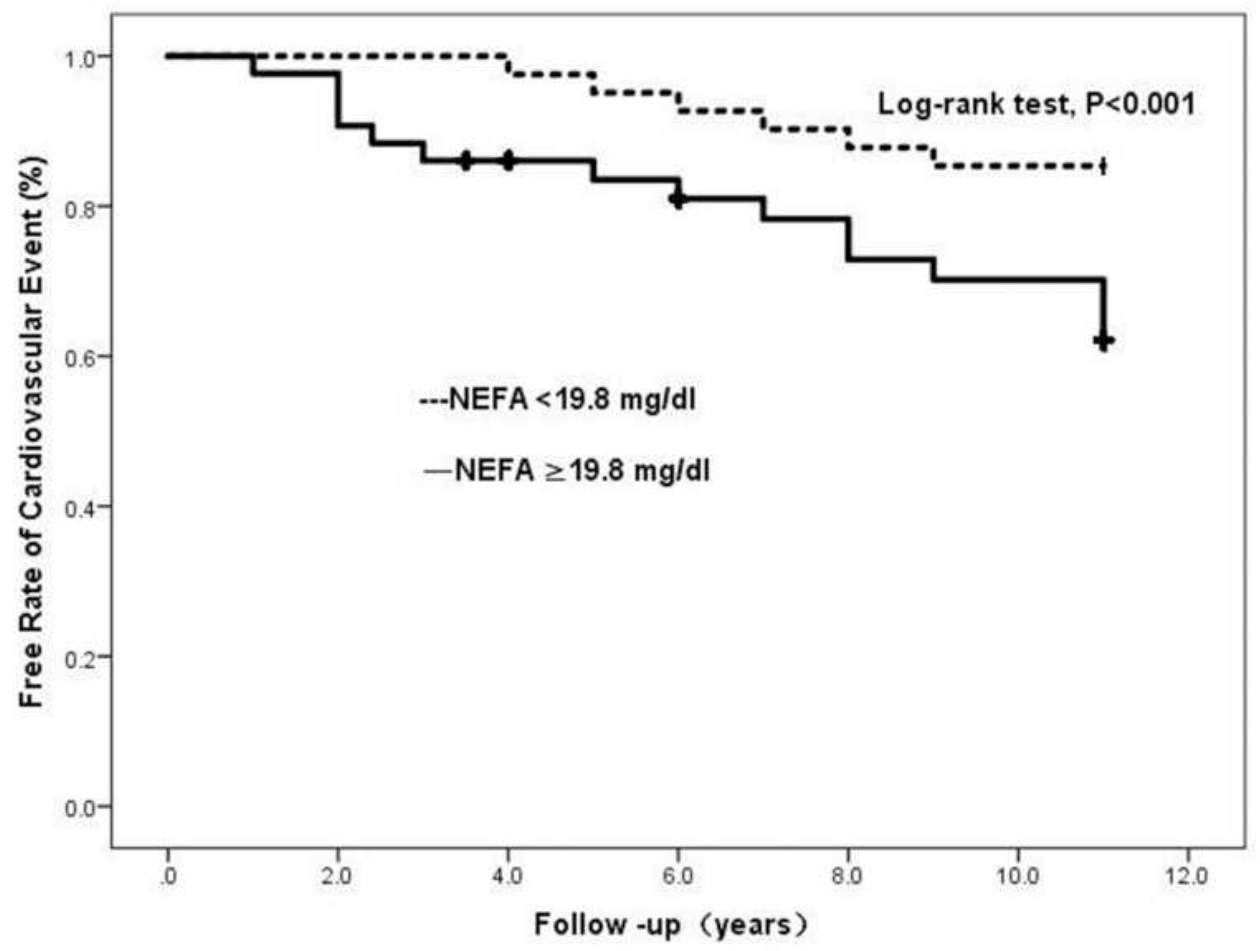

Figure I Kaplan-Meier analysis of cardiovascular events free stratified into 2 groups by the median levels of serum nonesterified fatty acid (NEFA).

95\% CI 1.34-2.20, P-trend $<0.001$, Model 3) after adjusting for these confounding factors (Table 3 ).

\section{Elevated Serum Levels of NEFA Were Independently Associated with Increased Risk of Cardiovascular Events in CKD Patients by Stratified Analysis}

For further confirmation of the independent association, whether or not the mass may be affected by "CVD history" and "obesity $(\mathrm{BMI} \geq 28)$ ", respectively. The stratified analyses by, respectively, adding "CVD history" and "obesity (BMI $\geq 28)$ " as covariates were performed. Our results suggested that serum NEFA levels were independently associated with increased risk of cardiovascular events $(\mathrm{HR}=1.61,95 \%$ CI $1.28-2.78, P=0.014)$ in the CKD patients with CVD history, but not in those without CVD history (HR=1.16, 95\% CI 0.90-1.58, $P=0.241)$ in Table 4, which implied that the independent association may affect by CVD history. Additionally, we also found that the independent association between serum NEFA levels and risk of cardiovascular events can be affected obesity (BMI $\geq 28)$ in Table 5 .

\section{Discussion}

We first found evidence that serum NEFA levels were related to a composite cardiovascular event in subjects with CKD in a prospective cohort study. We showed that

Table 2 Multivariate Cox Regression Analysis of Predicting Cardiovascular Events in 957 Patients with CKD

\begin{tabular}{|l|c|c|c|}
\hline Serum NEFA Levels & Model I & Model 2 & Model 3 \\
\hline Quartile I (low) & 1.000 (ref.) & 1.000 (ref.) & 1.000 (ref.) \\
Quartile 2 & $1.53(1.34-1.87)$ & $1.47(1.29-1.84)$ & $1.43(1.25-1.76)$ \\
Quartile 3 & $1.71(1.42-2.19)$ & $1.58(1.37-2.10)$ & $1.54(1.34-2.02)$ \\
Quartile 4 (high) & $1.79(1.45-2.38)$ & $1.66(1.42-2.26)$ & $1.62(1.40-2.16)$ \\
P-trend & $<0.001$ & $<0.001$ & $<0.001$ \\
\hline
\end{tabular}

Notes: Model I: Adjusted for age and gender. Model 2: Adjusted for age, gender, BMI, current smoker, current drinker, systolic and diastolic BP and regular physical activity. Model 3: Adjusted for age, gender, BMI, current smoker, current drinker, systolic and diastolic BP, regular physical activity, stage of CKD, laboratory indexes and medications. Abbreviations: CKD, chronic kidney disease; BMI, body mass index; BP, blood pressure. 
Table 3 Sensitivity Analysis of Predicting Cardiovascular Events in 957 Patients with CKD

\begin{tabular}{|l|c|c|c|}
\hline Serum NEFA Levels & Model I & Model 2 & Model 3 \\
\hline Quartile I (low) & 1.000 (ref.) & 1.000 (ref.) & 1.000 (ref.) \\
Quartile 2 & $1.43(1.32-1.84)$ & $1.36(1.29-1.80)$ & $1.34(1.24-1.75)$ \\
Quartile 3 & $1.49(1.36-1.98)$ & $1.47(1.32-1.87)$ & $1.38(1.29-1.83)$ \\
Quartile 4 (high) & $1.54(1.38-2.46)$ & $1.49(1.36-2.25)$ & $1.46(1.34-2.20)$ \\
P-trend & $<0.001$ & $<0.001$ & $<0.001$ \\
\hline
\end{tabular}

Notes: Model I: Adjusted for age, gender and dialysis treatment. Model 2: Adjusted for age, gender, BMI, current smoker, current drinker, systolic and diastolic BP, regular physical activity and dialysis treatment. Model 3: Adjusted for age, gender, BMI, current smoker, current drinker, systolic and diastolic BP, regular physical activity, stage of CKD, laboratory indexes, medications and dialysis treatment.

Abbreviations: CKD, chronic kidney disease; BMI, body mass index; BP, blood pressure.

Table 4 Stratified Analysis of Predicting Cardiovascular Events in 957 Patients with CKD

\begin{tabular}{|l|c|c|c|}
\hline Serum NEFA Levels & Model I & Model 2 & Model 3 \\
\hline CVD history (Yes) & $1.68(1.4 I-3.14)$ & $1.64(1.36-2.92)$ & $1.61(1.28-2.78)$ \\
P value & 0.008 & 0.010 & 0.014 \\
\hline CVD history (No) & $1.26(1.02-1.84)$ & $1.19(0.94-1.67)$ & $1.16(0.90-1.58)$ \\
P value & 0.040 & 0.082 & 0.241 \\
\hline
\end{tabular}

Note: Adjusted for age, gender, BMI, current smoker, current drinker, systolic and diastolic BP, regular physical activity, stage of CKD, laboratory indexes, medications and dialysis treatment.

Abbreviations: CKD, chronic kidney disease; BMI, body mass index; BP, blood pressure.

serum NEFA may be considered as a potential biomarker on detecting a composite cardiovascular event, including myocardial infarction, ischemic and/or hemorrhagic stroke, worsening heart failure, peripheral vascular diseases and sudden cardiac death.

NEFA is derived from the hydrolysis of triglycerides in adipose tissue and is an important energy supply for body. ${ }^{10,11}$ Serum NEFA levels increase in some conditions including hypertension, dyslipidemia, obesity and presence of diabetes in non-CKD studies ${ }^{23}$ as well as in our results in a CKD population. Here, we showed that serum NEFA can predict cardiovascular events in a large population with CKD. This is in line with previous cohort study with a significant association between NEFA and cardiovascular mortality in old individuals with $\mathrm{CKD} .{ }^{24}$ Another study has been to find that serum NEFA could predict all-cause mortality. ${ }^{15}$ Moreover, NEFA was also associated with risk of acute myocardial infarction ${ }^{14-17}$ and the risk of sudden cardiac death, ${ }^{16,17}$ which is also consistent with results. However, some observational studies did not find significant association. ${ }^{18}$ For instance, the Paris Prospective Study failed to observe an association between NEFA and acute myocardial infarction. ${ }^{18}$ The difference in results may be explained by different populations selected, different study designs, the different hypotheses investigated and different confounding factors adjusted. It is well known that NEFA is the energy source for myocardial energy metabolism. ${ }^{25,26}$ Our results on a potentially causal link between NEFA and cardiovascular events may be explained by several mechanisms: 1) The NEFA on cardiovascular toxicity has damage in

Table 5 Stratified Analysis of Predicting Cardiovascular Events in 957 Patients with CKD

\begin{tabular}{|c|c|c|c|}
\hline Serum NEFA Levels & Model I & Model 2 & Model 3 \\
\hline Obesity (BMI $\geq 28$ ) & $1.79(1.35-2.85)$ & $1.74(1.32-2.77)$ & $1.72(|.3|-2.7 \mid)$ \\
\hline$P$ value & 0.003 & 0.005 & 0.006 \\
\hline Obesity $(\mathrm{BMI}<28)$ & $1.08(0.96-1.89)$ & $1.06(0.94-1.8 I)$ & $1.05(0.92-1.79)$ \\
\hline$P$ value & 0.108 & 0.226 & 0.235 \\
\hline
\end{tabular}

Note: Adjusted for age, gender, BMI, current smoker, current drinker, systolic and diastolic BP, regular physical activity, stage of CKD, laboratory indexes, medications and dialysis treatment.

Abbreviations: CKD, chronic kidney disease; BMI, body mass index; BP, blood pressure. 
mitochondrial function and further leads to damage in plasma membranes, which may pose proarrhythmic effects; ${ }^{27-29} 2$ ) Elevated NEFA levels and increased b-oxidation in the myocardial tissue can be harmful for cardiovascular tissues through increased oxidative stress ${ }^{25}$ and activated apoptosis of endothelial cells. ${ }^{30}$ These pathological processes play an important role in the occurrence of cardiovascular events.

Notable strengths of our study included a large-sample cohort with a long follow-up period and detailed clinical characterization. $^{31}$ On the one hand, we first found that serum NEFA might be used as a value predictor for cardiovascular events in patients with CKD. On the other hand, we ensured comprehensive follow-up and rigorous adjudication of cardiovascular events. Sufficient confounding factors were corrected for the association between serum NEFA and cardiovascular events. Limitations did also exist. For example, we assessed eGFR and stage of CKD based on CKD-EPI creatinine. Although CKD-EPI creatinine is commonly used in most clinical studies, CKD misclassification or variations of eGFR levels may lead to some potential bias in results. We do not know whether changes of NEFA levels over the follow-up period because NEFA was tested only once at baseline.

\section{Conclusions}

Serum NEFA was related to with increased risk of cardiovascular events in patients with CKD. Our findings may provide an interesting perspective focusing on improved CVDs via reduced NEFA levels.

\section{Funding}

There is no funding to report.

\section{Disclosure}

The authors report no conflicts of interest in this work.

\section{References}

1. Coresh J, Selvin E, Stevens LA, et al. Prevalence of chronic kidney disease in the United States. JAMA. 2007;298(17):2038. doi:10.1001/ jama.298.17.2038

2. Levey AS, Eckardt KU, Tsukamoto Y, et al. Definition and classification of chronic kidney disease: a position statement from kidney disease: improving global outcomes (KDIGO). Kidney Int. 2005;67 (6):2089-2100. doi:10.1111/j.1523-1755.2005.00365.x

3. Foundation, National Kidney. KDOQI clinical practice guidelines and clinical practice recommendations for diabetes and chronic kidney disease. Am J Kidney Dis. 2006;47(3):11-145.

4. Herzog CA, Asinger RW, Berger AK, et al. Cardiovascular disease in chronic kidney disease. A clinical update from kidney disease: improving global outcomes (KDIGO). Kidney Int. 2011;80(6):572-586. doi:10.1038/ki.2011.223
5. Stenvinkel P. Chronic kidney disease: a public health priority and harbinger of premature cardiovascular disease. J Intern Med. 2010;268(5):456-467. doi:10.1111/j.1365-2796.2010.02269.x

6. Christa M, Angela D, Hannelore L. Chronic kidney disease and risk of incident myocardial infarction and all-cause and cardiovascular disease mortality in middle-aged men and women from the general population. Eur Heart J. 2006;(10):1245.

7. Cachofeiro V, Goicochea M, de Vinuesa SG, Oubiña P, Lahera V, Luño J. Oxidative stress and inflammation, a link between chronic kidney disease and cardiovascular disease. Kidney Int Suppl. 2008;74 (Suppl. 111):S4-S9. doi:10.1038/ki.2008.516

8. Ruiz S, Pergola PE, Zager RA, et al. Targeting the transcription factor Nrf2 to ameliorate oxidative stress and inflammation in chronic kidney disease. Kidney Int. 2013;83(6):1029-1041. doi:10.1038/ ki.2012.439

9. Kim HJ, Vaziri ND. Contribution of impaired Nrf2-Keap1 pathway to oxidative stress and inflammation in chronic renal failure. $\mathrm{Am}$ J Physiol Renal Physiol. 2010;298(3):F662-71. doi:10.1152/ ajprenal.00421.2009

10. Stich V, Berlan M. Physiological regulation of NEFA availability: lipolysis pathway. Proc Nutr Soc. 2004;63(2):369-374. doi:10.1079/ PNS2004350

11. Pilz S, März W. Free fatty acids as a cardiovascular risk factor. Clin Chem Lab Med. 2008;46(4):429-434. doi:10.1515/CCLM.2008.118

12. Opie LH, Knuuti J. The adrenergic-fatty acid load in heart failure. $J$ Am Coll Cardiol. 2009;54(18):1637-1646. doi:10.1016/j. jacc.2009.07.024

13. Boden G. Role of fatty acids in the pathogenesis of insulin resistance and NIDDM. Diabetes. 1997;46(1):3. doi:10.2337/diab.46.1.3

14. Roy VK, Kumar A, Joshi P, et al. Plasma free fatty acid concentrations as a marker for acute myocardial infarction. $J$ Clin Diagn Res. 2013;7(11):2432-2434.

15. Pilz S, Scharnag H, Tiran B, et al. Mo-P1:118 free fatty acids are independently associated with all-cause and cardiovascular mortality in subjects with coronary artery disease. Atheroscler Suppl. 2006;7 (3):72.

16. Pilz S, Scharnagl H, Tiran B. Elevated plasma free fatty acids predict sudden cardiac death: a 6.85-year follow-up of 3315 patients after coronary angiography. Eur Heart J. 2007;28(22):2763-2769. doi:10.1093/eurheartj/ehm343

17. Havmoeller R, Reinier K, Teodorescu C, et al. Elevated plasma free fatty acids are associated with sudden death: a prospective community-based evaluation at the time of cardiac arrest. Heart Rhythm. 2014;11(4):691-696. doi:10.1016/j.hrthm.2014. 01.012

18. Jouven X, Charles MA, Desnos M, et al. Circulating nonesterified fatty acid level as a predictive risk factor for sudden death in the population. Circulation. 2001;104(7):756-761. doi:10.1161/ hc3201.094151

19. Marie AC, Annick F, Nadine T, et al. High plasma nonesterified fatty acids are predictive of cancer mortality but not of coronary heart disease mortality: results from the Paris Prospective Study. Am J Epidemiol. 2001;153(3):292-298.

20. Pirro M, Mauriège $P$, Tchernof A. Plasma free fatty acid levels and the risk of ischemic heart disease in men: prospective results from the Québec Cardiovascular Study. Atherosclerosis. 2002;160 (2):377-384. doi:10.1016/S0021-9150(01)00588-3

21. Coresh J, Levey AS, Levin A, et al. Chronic kidney disease definition A stable definition of chronic kidney disease improves knowledge and patient care. BMJ. 2013;347(sep18 1):f5553. doi:10.1136/bmj. f5553

22. Stevens PE. Evaluation and management of chronic kidney disease synopsis of the kidney disease: improving global outcomes 2012 clinical practice guideline. Ann Intern Med. 2013;158(11):825. doi:10.7326/0003-4819-158-11-201306040-00007 
23. Mozaffarian D. Free fatty acids, cardiovascular mortality, and cardiometabolic stress. Eur Heart J. 2007;28(22):2699-2700. doi:10.1093/ eurheartj/ehm 451

24. Zibo X, Xu H, Huang X. Nonesterified fatty acids and cardiovascular mortality in elderly men with CKD. Clin J Am Soc Nephrol. 2015;10 (4):584-591. doi:10.2215/CJN.08830914

25. Wu Y, Zhou H, Wu K, et al. PTEN phosphorylation and nuclear export mediate free fatty acid-induced oxidative stress. Antioxid Redox Signal. 2014;20(9):1382-1395. doi:10.1089/ars.2013.5498

26. Boden G. Obesity, insulin resistance and free fatty acids. Curr Opin Endocrinol Diabetes Obes. 2011;18(2):139-143. doi:10.1097/ MED.0b013e3283444b09

27. Oliver MF. Sudden cardiac death: the lost fatty acid hypothesis. QJM. 2006;99(10):701. doi:10.1093/qjmed/hcl084
28. Makiguchi M, Kawaguchi H, Tamura M, et al. Effect of palmitic acid and fatty acid binding protein on ventricular fibrillation threshold in the perfused rat heart. Cardiovasc Drugs Ther. 1991;5(4):753-761. doi:10.1007/BF03029751

29. Soloff LA. Arrhythmias following infusions of fatty acids. Am Heart J. 1970;80(5):671-674. doi:10.1016/0002-8703(70)90012-8

30. Shimabukuro M. [Insulin resistance and free fatty acid]. Nihon Rinsho. 2012;70(Suppl3):185-190. Japanese.

31. Djousse L, Biggs ML, Ix JH, et al. Nonesterified fatty acids and risk of sudden cardiac death in older adults. Circ Arrhythm Electrophysiol. 2012;5(2):273. doi:10.1161/CIRCEP.111.967661

\section{Publish your work in this journal}

The International Journal of General Medicine is an international, peer-reviewed open-access journal that focuses on general and internal medicine, pathogenesis, epidemiology, diagnosis, monitoring and treatment protocols. The journal is characterized by the rapid reporting of reviews, original research and clinical studies across all disease areas. The manuscript management system is completely online and includes a very quick and fair peer-review system, which is all easy to use. Visit http://www.dovepress.com/ testimonials.php to read real quotes from published authors. 